\title{
Le contexte faiseur et défaiseur de figures, ou la conditionnalité de la reconnaissance figurale
}

How Context does and undoes Figures of Speech or the Conditional Nature of the Recognition of Figurative Speech

\section{Anna Jaubert}

\section{OpenEdition}

Journals

Édition électronique

URL : http://journals.openedition.org/pratiques/2452

DOI : 10.4000/pratiques.2452

ISSN : 2425-2042

Éditeur

Centre de recherche sur les médiations (CREM)

Référence électronique

Anna Jaubert, «Le contexte faiseur et défaiseur de figures, ou la conditionnalité de la reconnaissance figurale », Pratiques [En ligne], 165-166 | 2015, mis en ligne le 01 octobre 2015, consulté le 03 mai 2019. URL : http://journals.openedition.org/pratiques/2452 ; DOI : 10.4000/pratiques.2452

Ce document a été généré automatiquement le 3 mai 2019.

(c) Tous droits réservés 


\section{Le contexte faiseur et défaiseur de figures, ou la conditionnalité de la reconnaissance figurale}

How Context does and undoes Figures of Speech or the Conditional Nature of the Recognition of Figurative Speech

Anna Jaubert

1 Si pour C. S. Du Marsais (1988: 35), les «manières de parler » sont d'abord des manières de penser, c'est probablement que ces manières de parler sont de l'appropriation du langage (Jaubert, 2007) : appropriation d'un sujet en situation à son ressenti des choses, et à son intention de le communiquer. "On ne peut comprendre le langage si l'on ne comprend pas le discours, et l'on ne peut comprendre le discours si l'on ne prend pas en considération le but de la communication ». Cet axiome de P. F. Strawson (1970) qui soustend l'analyse pragmatique des énoncés est applicable au langage figuré, qui est du langage même, du plus spontané au plus sophistiqué. S'agissant des figures dites «de pensée ", "macrostructurales " pour G. Molinié, on sait qu'elles ne se signalent pas de soi, mais qu'elles exigent la confrontation d'une forme linguistique et de son contexte. Dans la perspective de la pragmatique des figures de M. Bonhomme (2005) et de ma lecture pragmatique des postures énonciatives, l'identification des figures déclenchée par des phénomènes de saillance linguistique, ne s'achève qu'à la faveur de l'acte de langage qu'elles réalisent. Les conditions de production et de réception qui déterminent l'interprétation d'un discours, pèsent sur la reconnaissance des figures qu'on y rencontre. Nous verrons comment le contexte non seulement fait les figures, mais comment parfois il les défait.

2 Pour expliquer un tel processus, je reviendrai sur le constat que les figures résistent parfois aux typologies. Ce n'est pas manque de perspicacité de l'observateur, c'est la conséquence de la conditionnalité fondamentale des figures "de pensée ", irréductiblement dépendantes de leur environnement. On se tournera alors vers l'analyse 
énonciativopragmatique, et l'approche positive de la problématicité figurale. Pour finir, j'illustrerai le rôle décisif du contexte dans la pertinentisation du sens figuré, comme dans son éventuelle déprogrammation.

\section{L'insécurité typologique}

3 Pourquoi les figures de pensée défient-elles les typologies? Malgré l'inflation taxinomique, «la rage de nommer », soulignée par G. Genette (1962: 214; $1977: 16)$, la rhétorique, qui est une construction de la parole efficace, ne peut se réduire à une collection de procédés discrets ${ }^{1}$. Même si les figures, en tant que formes marquées, présentent des structures caractéristiques suffisamment stables pour être codifiées et donner lieu à toute une nomenclature (et quelle nomenclature!), leur fonctionnalité, c'est-à-dire le rendement qu'elles confèrent à l'énoncé (Bonhomme, 2005: 153-201), dépend étroitement de leur contexte d'apparition. Or, les déterminations contextuelles sont nombreuses, et variables. Les figures de pensée, définies comme ne se signalant pas de soi, et mobilisant des empans de discours plus ou moins longs, résultent de la confrontation entre une forme linguistique et un environnement dont la prégnance s'exerce à plusieurs niveaux. Nous verrons en III que le cotexte immédiat peut entrer en conflit avec un contexte élargi. La problématicité de la reconnaissance tient à la multiplicité et aux interférences des données contextuelles: repères culturels inégalement partagés, évolutifs dans le temps, situation d'énonciation qui peut être inédite, état de la langue, dispositions psychologiques du locuteur, etc. La part, considérable ici, de la compétence encyclopédique, explique la possible dissymétrie entre l'encodage et le décodage. On comprend que l'hésitation des étiquettes reflète la difficulté, sur le terrain, à identifier sans reste une stratégie dont les causes comme les effets sont souvent stratifiés.

4 Les figures hybrides offrent un bon exemple de remise en question typologique. Si nous envisageons le cas, souvent débattu, de l'euphémisme et de la litote (Jaubert, 2008, 2011), l'opposition est claire en théorie, mais en pratique ces figures sont parfois difficiles à démêler. S'employant à cerner la figuralité instable du "discours en deçà ", M. Bonhomme évoque les mixtes litote euphémique et euphémisme litotique.

5 Alors que l'euphémisme projette une atténuation parvenant à son terme, souvent par la voie directe d'un subterfuge lexical ${ }^{2}$, la litote pour sa part projette une atténuation feinte, et qui montre sa feinte par le choix d'une formulation qui tourne autour du pot. Le processus minorant s'en trouve non seulement enrayé, mais inversé (Jaubert, 2011). Les visées pragmatiques sont alors divergentes : dire moins pour minimiser le sujet qui fâche, ou au contraire dire moins pour faire entendre plus. Or, il arrive que cette divergence bien admise se dérobe sous des formes communes. Des énoncés comme « Vous n'ignorez pas que ", ou "Vous n'êtes pas sans savoir que ", se signalent par la négation du terme marqué qui affiche le caractère alambiqué de la double négation : on y reconnait la forme canonique de la litote. Leur visée cependant peut passer pour euphémique : lexicalisés dans le contexte d'un échange poli, ils se substituent au trop vif, et trop polémique « Vous savez pertinemment que... ». Au final, le dernier mot de la litote euphémique sera à la perception (l'uptake) du récepteur: sera-t-il plus réceptif à la conventionalisation courtoise, c'est-à-dire à l'euphémisme, ou à l'idée du plus, imputable à la litote ? Un même arbitrage vaut pour l'euphémisme litotique. Si le voile de la substitution euphémique, non conventionalisée cette fois, ressemble trop à un abus de langage, l'euphémisme bascule dans une réception litotique. C'est ce qu'on observe avec la réplique de Figaro : 
(1) «Je cherche dans ma tête si la pharmacie ne fournirait pas quelques petits

moyens innocents » (Beaumarchais, Le Barbier de Séville, Acte I, 4)

6 Les " petits moyens innocents ", au voisinage de la " pharmacie », ne disent rien moins que le projet joyeusement cynique de droguer toute la maisonnée de Bartholo. La réplique du comte, "Scélérat!", valide la visée litotique en poursuivant le jeu de la duplicité énonciative ${ }^{3}$.

Une autre différenciation est parfois malaisée, c'est celle entre l'humour et l'ironie. Certains linguistes (voir Charaudeau, 2006) proposent de hiérarchiser les termes, faisant de l'humour une catégorie englobante, mais la question de leur spécificité demeure. A. Rabatel (2013) s'appuie sur la notion de sous-énonciation vs sur-énonciation pour rendre compte d'une posture énonciative dont intuitivement nous ressentons bien la différence: rire aux dépens de, ou rire avec, moquerie connivente de l'humour ou raillerie clivante de l'ironie. Mais là encore sur le terrain on peut hésiter : dans l'exercice de la dérision les critères qui font pencher en faveur d'une figure ou d'une autre ne forment pas toujours des faisceaux stables. D. Mayaffre et moi-même avons observé l'impact de l'humour et de l'ironie dans la construction de l'ethos de François Hollande candidat à l'élection présidentielle (Jaubert \& Mayaffre, 2013). Le contexte est ici particulièrement contraignant: une élection uninominale au suffrage universel doit répondre à des attentes divergentes, flatter l'idéal de pugnacité des uns, l'idéal d'intelligence des autres, et même l'idéal d'une majorité qui voudrait les combiner ! Élection à deux tours, elle impose de ferrailler d'abord et de se réconcilier ensuite : d'éliminer des concurrents sans interdire leur ralliement. La dérision doit s'adapter à de subtils dosages qui relativisent l'opposition théorique humour vs ironie, pratiquant une ironie amortie pour un adversaire récupérable comme François Bayrou, ou glissant insensiblement de l'ironie à l'humour face à un public de sympathisants :

(2) «Certains me reprochent de n'avoir jamais été ministre. Quand je vois ceux qui le sont aujourd'hui, ça me rassure! Et ce sont les mêmes qui reprochaient en son temps à François Mitterrand de l'avoir été onze fois. Et dois-je rappeler, en gardant la comparaison, que Georges Clemenceau ne devint ministre et président du Conseil qu'à 65 ans? Mais je n'attendrai pas jusque là, je vous le promets " (discours prononcé au Bourget, le 22 janvier 2012).

C'est par une approche énonciativopragmatique que nous analyserons comment précisément le contexte joue son rôle de « levier figural ».

\section{L'approche énonciativopragmatique}

9 Nous venons de voir qu'entre certaines figures la frontière était franchissable. L'incertitude interprétative va plus loin: elle peut concerner l'existence même d'une acception figurée.

Je ne m'attarderai pas sur les mécomptes de l'ironie ou de l'humour, loin d'être toujours reçus « 5 sur 5 ». Selon l'appréciation du contexte, l'acte figural se réalise, ou pas.

11 L'hyperbole elle-même est moins assurée qu'on pourrait le croire, car le changement d'échelle des objets concernés est parfois motivé, inscrivant la démesure dans la normalité d'un nouvel univers. M. Bonhomme (1998: 76) observe que si Gargantua a été allaité par « dix et sept mille neuf cent treize vaches », cet énoncé n'est pas figuré une fois que la fiction nous a installés dans le monde des Géants. Je ferai la même remarque pour Micromégas, l'habitant de Sirius à qui Voltaire confie (justement!) la défense et 
illustration de la relativité. Selon le monde de référence, les proportions gigantesques se trouvent normalisées.

En matière de construction figurale, on voit se profiler ici le rôle des points de vue $e^{4}$ L'analyse énonciativopragmatique prend en compte le jeu des points de vue concentré par l'énonciation, un « dialogisme figural » (Calas et al., 2012).

13 J'ai pour ma part souvent fait appel au concept d'énonciation clivée pour expliquer le fonctionnement de certaines figures (Jaubert, 2008, 2011, 2013). L'énonciation clivée est celle qui fait entendre, ou simplement laisse entendre, une altérité. Dans le cas du discours figuré, la dualité n'est pas celle d'une autre voix plus ou moins actualisée (comme dans le discours rapporté), mais la confrontation intériorisée de deux points de vue, confrontation éminemment dissymétrique ${ }^{5}$. Ainsi s'explique le fonctionnement de l'ironie, qui projette une altérité pour la discréditer, de l'humour, qui est une cohabitation plus douce, de la litote, où la tension dialogique réveille l'idée du plus, de l'hyperbole, identifiable comme figure dans la mesure où l'énonciation clivée maintient le point de vue doxique. Quant à l'euphémisme, d'après les traités son statut de figure parait plus incertain: à mes yeux c'est précisément parce que la tension dialogique y est dépassée (Jaubert, 2008). Le point de vue de l'autre est celui d'un interdiscours assez consensuel pour recouvrir le point de vue de l'un.

14 La question qui se pose est donc celle de la problématicité attachée à l'énonciation rhétorique (Jaubert, 2011, 2013), qui produit une opacification fugitive, et parfois durable. Elle nous renvoie à la détermination contextuelle de la reconnaissance des figures.

\section{Quel contexte pour quelles figures ? Variabilité et stratification de l'environnement considéré}

15 «Produire des figures, c'est activer des schèmes discursifs qui modélisent certaines variations de la langue en vue d'augmenter la pertinence de la communication" (Bonhomme, 2005: 41). La reconnaissance de l'acte figural suppose donc celle de sa pertinence communicationnelle, autrement dit, elle résulte d'un rapport lisible entre le choix d'une forme particulière et du contexte où elle apparait. Si, dans la coconstruction envisagée, une pertinentisation se dessine (voir Du Marsais sur l'hyperbole), en contrepartie une déprogrammation aussi est possible.

Lors d'un colloque récent sur l'hyperbole (Berne, 5-6 septembre 2013), plusieurs intervenants ont souligné sa figuralité conditionnelle. Cette figure en effet mobilise la confrontation de l'intensité à un « sens commun " plus ou moins stabilisé, historiquement et idéologiquement, plus ou moins attendu dans le genre de discours au sein duquel l'énoncé fait sens. Hyperbole pour qui, et dans quel univers esthétique, socioculturel, affectif... ? Il apparait qu'en contexte politique (mais encore faudrait-il y distinguer différentes scènes énonciatives), le discours intensif, hyperbolique au sens large (Mayaffre, 2012), ne relève pas de l'énonciation clivée commune aux figures "par réflexion » (Fontanier, 1977 : 123-124), énonciation clivée qui elle-même prédispose une adhésion clivée, mais qu'il se revendique comme le discours intensif d'une personnalité « de conviction » qui requiert l'adhésion tout court.

17 Pour la litote, l'instruction linguistique livrée par sa forme négative canonique, que j'ai appelée le « lit de la litote » (Jaubert, 2011 : 155), est celle d'une inversion qui promeut la réinterprétation de l'énoncé à la hausse, parfois même sans l'appui du contexte (d'où la 
lexicalisation fréquente des formules litotiques). Contrairement à ce que l'on pourrait croire, la lecture figurale n'est pas une lecture difficilior, c'est souvent la lecture la plus spontanée. C'est bien ce qui s'est produit avec la réception de la réplique de Chimène «Va, je ne te hais point », célébrée depuis Du Marsais comme une litote, mais qu’à la suite de L. Marquèze-Pouey (1973), et pour développer une intuition où se rejoignaient G. Molinié (1992) et G. Forestier (1993), j'ai rendu à une lecture littérale, beaucoup plus cohérente dans le cadre de l'interaction où elle se situe (Jaubert, 2008, 2011), à savoir : une réfutation dans le cotexte immédiat de l'enchainement, une argumentation pour apaiser Rodrigue (qui demande à Chimène de le tuer), et un appel à la gloire très cornéliens dans l'ensemble de la scène, où Chimène, loin de masquer son amour, le revendique au contraire comme faisant toute la "générosité ", c'est-à-dire tout l'héroïsme de sa conduite :

(3) « De quoi qu'en ta faveur notre amour m'entretienne

Ma générosité doit répondre à la tienne

[...] je veux que la voix de la plus noire envie

Élève au ciel ma gloire et plaigne mes ennuis,

Sachant que je t'adore et que je te poursuis » (Corneille, Le Cid, III, 4, v. 929-930;

v. 970-972).

La cause parait donc entendue lorsqu'on considère le contexte local de la scène et de l'œuvre, sans oublier surtout le cotexte-même de l'enchainement ${ }^{6}$ :

(4) « Rodrigue. - Ton malheureux amant aura bien moins de peine

A mourir de ta main, qu'à vivre avec ta haine.

Chimène. - Va, je ne te hais point » (ibid., v. 961-963)

où le « Va » fonctionne comme un marqueur interlocutif de reprise conciliante, visant (un peu comme " allons/ allons, allons/ allez... ») à rassurer l'allocutaire, à dédramatiser son propos, en faisant réviser non pas à la hausse ce qu'on dit, mais à la baisse ce que l'autre vient de dire (à rapprocher des répliques en amont, v. 939-940: «Je mourrai trop heureux, mourant d'un coup si beau/ Va, je suis ta partie, et non pas ton bourreau »).

D'où vient qu'un siècle plus tard Du Marsais (1730) l'érige en litote exemplaire, sachant que cette interprétation était probablement déjà dans l'air du temps (on la retrouve dans Le Jeu de l'amour et du hasard joué pour la première fois en cette même année 1730), et qu'elle a eu la postérité qu'on sait. C'est que la citation ne vit pas une vie d'énoncé ordinaire. Dans ma démonstration de 2008, à la suite d'un échange avec A. Rabatel que je remercie, j'avais avancé l'hypothèse de la décontextualisation et de la recontextualisation possible de certains énoncés. Je reviens ici à cette hypothèse, en insistant sur la variabilité et la stratification du contexte.

21 La citation, parole exemplaire, court le risque de la décontextualisation. C'est le point important. Une formule sortie de son environnement discursif immédiat devient problématique; reversée alors dans un contexte plus large, elle se voit prêter une autre pertinence. Pensons aux polémiques qui entourent certaines petites phrases montées en épingle par les médias. Pour "Va, je ne te hais point», on a perdu de vue l'interaction précise de la scène, la psychologie du "généreux" cornélien, pour mobiliser une motivation socioculturelle plus diffuse où la pudeur imposée aux femmes, en convergence avec les valeurs de délicatesse promues dans les salons, ferait pressentir un " je t'aime » litotisé. Rappelons aussi que la lecture litotique est déclenchée par une instruction linguistique forte, et qu'il faut un cotexte très balisé pour la déprogrammer ${ }^{7}$. La réception littérale n'est donc pas forcément la plus spontanée... 
L'interprétation qui a prévalu et qui s'est répercutée d'un manuel de rhétorique à l'autre est le fait d'une contextualisation large qui l'a emporté sur le cotexte immédiat de la scène. Mais si l'on y réfléchit, on a d'autant plus facilement intégré les conventions mondaines de l'époque motivant un aveu retenu, que cette retenue elle-même entre en résonance avec une disposition psychologique qui transcende les époques. À ce niveau, la recontextualisation est donc plus large encore. Le fait est que la déclaration d'amour est un acte de langage couteux : ainsi, chez Racine, elle se présente comme une transgression de l'indicible qui précipite la catastrophe (Tamas, 2012), et, plus proche de nous, la formule «je t'aime» analysée par A. Finkielkraut (1976), montre à quel point sa profération est lourde d'enjeux... et donc bonne candidate à l'expression détensive de la litote $^{8}$. On observe ici que le périmètre contextuel influe sur la réception d'une figure, mais qu'il n'est pas un acquis définitif, et qu'une recontextualisation peut être promue par la pragmatique même de la figure. Alors la figure deviendrait contextualisante à son tour, et permettrait une double interprétation'.

En l'occurrence le contexte large existe donc, et ne peut être tenu pour négligeable ${ }^{10}$. Si au niveau de la réplique, il y a bien eu méprise, la méprise s'est acquis un droit de cité. Le contexte c'est aussi notre réception en feuilleté (Molinié, 1989) des figures. Depuis Du Marsais « Va je ne te hais point » est, qu'on le veuille ou non, la formule qui identifie la litote et forme un nouveau contexte culturel. Prolongeant mon analyse, il faudrait réfléchir aux différentes façons dont se résout le conflit possible entre cotexte immédiat et contexte large. Pour l'exemple qui nous occupe, une diction de l'actrice jouant le rôle de Chimène laisserait passer dans sa réfutation littérale la distanciation d'une présumée litote rebrodant ses déclarations antérieures ${ }^{11}$, une coquetterie après tout compatible avec l'esprit de la tragicomédie.

Figure à un niveau, réfutation littérale à un autre. Que pouvons-nous en conclure, provisoirement au moins? Il apparait que le rapport entre la figure et son contexte cristallise la problématicité du fonctionnement figural : la dissymétrie entre l'encodage et le décodage s'expliquant par l'inégalité des univers de croyance entre le locuteur et l'allocutaire. Le contexte à l'évidence n'est ni monolithique, ni immuable. Sa stratification et ses recompositions possibles légitiment une réception feuilletée des figures où la notion d'ambigüité fait place à celle de vérités successives.

\section{BIBLIOGRAPHIE}

BEAUZÉE, N., DU MARSAIS, C. S., MARMONTEL, J.-F. \& Voltaire (1784) : Encyclopédie Méthodique.

Grammaire et littérature, t. II, Paris, Panckoucke.

BONHOMme, M. (1998) : Les figures du discours, Paris, Éd. Le Seuil.

- (2005) : Pragmatique des figures du discours, Paris, H. Champion.

CAlas, F., FROMilhague, C., GARAgnon, A.-M. \& SUSini, L. (dirs) (2012) : Les Figures à l'épreuve du discours. Dialogisme et polyphonie Paris, Presses de l'Université Paris-Sorbonne. 
CHARAUdeau, P. (2006) : « Des catégories pour l'humour? », Questions de communication, 10, p. 19-41.

DUCRot, O. (1998) [1972] : Dire et ne pas dire. Principe de sémantique linguistique, Paris, Hermann. DU MARSAIS, C. S. (1988) [1730] : Traité des Tropes, Paris, Flammarion.

FinkielKRAUt, A. (1976) : «Sur la formule Je t'aime », Critique, 348, mai, p. 520-537.

FONTANIER, P. (1962) : « La rhétorique et l'espace du langage », Tel Quel, 11.

- (1977) : Les Figures du discours, Paris, Flammarion.

FORESTIER, G. (1993) : Introduction à l'analyse des textes classiques, Paris, Nathan.

GAUDIN-BORDES, L. \& SALVAN, G. (2013) : « Contextualisation et hyperpertinence figurale », Le Discours et la langue, t. 4.2, p. 17-24.

GENETTE, G. (1962) : « La rhétorique et l'espace du langage », Tel Quel, 11, p. 214.

- (1977) : «Introduction », in : P. Fontanier, Les Figures du discours, Paris, Flammarion, p. 5-17.

HORAK, A. (2011) : «Y a-t-il des litotes euphémiques ? ", in : A. Horak (dir.), La Litote. Hommage à Marc Bonhomme, Berne, P. Lang, p. 150-170.

JAUBERT, A. (1990) : La Lecture pragmatique, Paris, Hachette.

- (2007) : «La Diagonale du style », Pratiques, 135-136, déc., p. 47-62.

- (2008) : " Dire et plus ou moins dire. Analyse pragmatique de l'euphémisme et de la litote », Langue française, 160, p. 106-116.

- (2011) : « La litote est-elle un contre-euphémisme ? », in : A. Horak (dir.), La Litote. Hommage à Marc Bonhomme, Berne, P. Lang, p. 145-158.

- 2013, « La figure et le dess(e)in. Les conditions de l'acte ironique », L'Information grammaticale, 137, p. 29-35.

JAUBERT, A. \& MAYAFFRE, D. (2013) : «Ethos préalable et ethos (re)construit. La transformation de l'humour légendaire de François Hollande », Langage et Société, 146, p. 71-88.

KERBRAT-ORECCHIONI, C. (2011) : « Quand dire moins, c'est faire plus : une approche pragmatique de la litote », in : A. Horak (dir.), La Litote. Hommage à Marc Bonhomme, Berne, P. Lang, p. 67-97.

MARQUÈZE-POUEY, L. (1973) : « Va, je ne te hais point », Grammatica, II, p. 3-15.

MAYAFFRE, D. (2012) : Mesure et démesure du discours. Nicolas Sarkozy 2007-2012, Paris, Presses de Sciences Po.

MOLINIÉ, G. (1989) : La Stylistique, Paris, Presses universitaires de France.

- (1992) : Dictionnaire de rhétorique, Paris, Le Livre de poche.

RABATEL, A. (dir.) (2008) : « Figures et point de vue », Langue française, 160.

- (2013) : « Humour et sous-énonciation (vs ironie et sur-énonciation) », L'Information grammaticale, 137, mars, p. 36-42.

STRAWSON, P. F. (1970) : « Phrase et acte de parole », Langages, 17, p. 19-33.

TAMAS, J. (2012) : Dire et ne pas dire. Du silence éloquent à l'énonciation tragique des déclarations d'amour chez Racine, thèse, Université Paris-Sorbonne, 29 oct. 


\section{NOTES}

1. Comme les dictionnaires de rhétorique pourraient en entretenir l'illusion.

2. Beauzée et al. (1784: 39). Notons que, pour réussir son effet perlocutoire, ce subterfuge doit avoir l'air « naturel » (Bonhomme, 1998, 2005).

3. Voir infra le rôle de la cohabitation des points de vue.

4. Voir le cadre proposé par A. Rabatel (2008).

5. Dont A. Rabatel (2013 : 36-42) rend compte avec les notions de sur-énonciation et de sousénonciation.

6. Le cotexte antérieur dont C. Kerbrat-Orecchioni (2011: 82) relève également qu'il bloque l'interprétation litotique.

7. Comme dans le «testament spirituel » de Voltaire : « Je meurs en adorant Dieu, en aimant mes amis, en ne haïssant pas mes ennemis, en détestant la superstition ».

8. O. Ducrot (1998) rappelle qu'il y a "au moins une condition nécessaire à l'interprétation litotique, c'est que certaines raisons (peut-être des conventions sociales) s'opposent, dans la situation de discours donnée, à l'emploi d'un énoncé plus fort ».

9. Voir C. Kerbrat-Orecchioni (2011), L. Gaudin-Bordes et G. Salvan (2013).

10. Ajoutons que la formule, consacrée litote par la tradition critique, assure en tant que telle bien des clins d'œil intertextuels au théâtre (par exemple l'aveu de Silvia dans Le Jeu de l'amour et du hasard, ou l'aveu de Célimène dans le pastiche de Jacques Rampal Célimène et le Cardinal, Paris, Librairie théâtrale, 1993).

11. N'oublions pas que dans cette scène Chimène a déjà reconnu son amour trois fois, et qu'elle ne se privera pas de le répéter.

\section{RÉSUMÉS}

«On ne peut comprendre le langage si l'on ne comprend pas le discours et l'on ne peut comprendre le discours si l'on ne prend pas en considération le but de la communication » : cet axiome de P.F. Strawson qui sous-tend l'analyse pragmatique de nos productions verbales s'applique évidemment au langage figuré. S'agissant des figures dites "de pensée " dans la rhétorique classique, ou figures macrostructurales (Molinié, 1992), on sait depuis longtemps qu'elles ne se signalent pas de soi, mais qu'elles exigent la confrontation d'une forme linguistique et d'un contexte. Pour une approche pragmatique, ces figures sont des comportements énonciatifs et leur identification ne s'achève qu'à la faveur de l'acte de langage qu'elles réalisent. L'ironie n'est reconnue comme telle que si l'on perçoit l'intention moqueuse qui la motive, et, le parler intensif ne produit pas toujours une hyperbole. Les conditions de production et de réception qui déterminent l'interprétation d'un discours, décident de sa figuralité. C'est donc le contexte qui au final fait les figures, ou les défait. On verra avec la litote que la lecture figurale n'est pas la plus couteuse, que le contexte au sens large peut entrer en conflit avec le cotexte immédiat, et qu'il faut alors une contrainte précise dans l'environnement discursif pour « déprogrammer » une figure qui, décantée dans notre imaginaire discursif, déclenche un réflexe d'interprétation de soi. 
"Language cannot be understood unless discourse is understood and discourse cannot be understood unless the aim of communication is taken into account". This axiom, which was formulated by P. F. Strawson, underlies the necessity for a pragmatic analysis of our verbal productions and can obviously be applied to figurative language. If one takes for instance the socalled "figures of thought" of classical rhetoric (what Georges Molinié (1992) calls "macrostructural figures"), it is well known that they are not meant to attract attention to themselves, since they depend upon a specific relationship between a signifying unit and its context. Seen from a pragmatic perspective, those figures appear to be so many enunciative types of behaviour that can only be identified if one takes the speech act which they realize into consideration. Irony is not recognized as such if one does not perceive the mocking intention that motivates it, and speaking vehemently does not always imply the use of hyperboles. The conditions of production and reception that determine the interpretation of discourse also determine its potential for figurative interpretation. Thus, all things considered, it is context that produces or prevents the emergence of figurative speech. I shall study a few occurrences of understatements, in order to demonstrate that it is easier to interpret them from a figurative point of view than in a literal manner, and also that context lato sensu may be incompatible with its immediate cotext. Finally, specific constraints in the verbal environment are needed to do away with a figure of speech which, once it has taken shape in our discursive imagination, triggers a reflex response of self-interpretation.

\section{INDEX}

Mots-clés : analyse pragmatique, acte de langage, figuralité conditionnelle, imaginaire discursif Keywords : pragmatic analysis, speech act, conditional nature of figurative speech, discursive imagination

\section{AUTEUR}

\section{ANNA JAUBERT}

BCL (UMR 7320 CNRS), Université Nice Sophia Antipolis 